\title{
Análisis bibliométrico de la investigación sobre tuberculosis en el Perú, periodo 1981-2010
}

Research on tuberculosis in Peru: bibliometric analysis 1981-2010

\author{
Martín Yagui-Moscoso ${ }^{1,2}$, Héctor Oswaldo-Jave ${ }^{3}$, Maricela Curisinche-Rojas², \\ César Gutiérrez ${ }^{1}$, Franco Romaní-Romani ${ }^{1,2}$ \\ 'Departamento Académico de Medicina Preventiva y Salud Pública, Universidad Nacional Mayor de San Marcos, Lima, Perú. \\ ${ }^{2}$ Instituto Nacional de Salud, Lima, Perú. \\ ${ }^{3}$ Hospital Nacional Dos de Mayo, Lima, Perú.
}

\begin{abstract}
Resumen
Introducción: La tuberculosis (TB) es una de las prioridades sanitarias y de investigación nacional. La producción científica sobre TB en el Perú no ha sido estudiada. Objetivo: Analizar las publicaciones sobre tuberculosis (TB) realizadas en el Perú en el periodo 1981 2010. Diseño: Estudio bibliométrico sobre tuberculosis en el Perú. Lugar: Universidad Nacional Mayor de San Marcos e Instituto Nacional de Salud, Lima, Perú. Unidad de análisis: Artículos originales, incluyendo reportes y series de casos, desarrollados completamente en Perú 0 estudios multicéntricos con participación de sedes peruanas. Intervenciones: La búsqueda bibliográfica fue realizada en revistas indizadas en Medline, SciELO y LILACS, entre 1981 y 2010. Para cada artículo se registró año de publicación, autores, duración de la ejecución, idioma, área de estudio, tipo de publicación, diseño de estudio, ámbito de estudio, población de estudio, tipo de investigación definida por OPS, instituciones participantes, fuentes de financiamiento y factor de impacto. Principales medidas de resultados: Indicadores bibliométricos de producción. Resultados: Identificamos 233 artículos sobre tuberculosis. El tiempo promedio de duración de los estudios fue de 2,79 $\pm 3,55$ años, mientras que el promedio entre la finalización del estudio y su publicación fue de 2,95 $\pm 1,65$ años. Respecto al área de estudio del artículo, 68 (29,2\%) trataron principalmente temas clínicos, $66(28,3 \%)$ el área de epidemiología, 56 (24\%) temas de salud pública. Solo 27,9\% de los artículos fue publicado en revistas biomédicas nacionales. La mayoría de artículos tuvo participación de alguna institución internacional (71\%). Conclusiones: A pesar del evidente incremento en la producción científica sobre TB en el Perú, esta aún se encuentra lejos de lo producido por países con mayor desarrollo. Además de adoptar medidas para incrementar la producción científica e investigación de un problema sanitario nacional como la TB, es necesario dirigir dicha investigación mediante una agenda nacional basada en un proceso de priorización, que la haga más efectiva.
\end{abstract}

Palabras clave: Tuberculosis, bibliometría, publicaciones científicas y técnicas, agenda de prioridades en salud.

Abstract

Background: Tuberculosis (TB) is sanitary and national research priority. Scientific production on TB in Peru has not been studied. Objectives: To analyze publications on tuberculosis (TB) done in Peru in the period 1981-2010. Design: Bibliometric study on tuberculosis in Peru. Setting: Universidad Nacional Mayor de San Marcos and Instituto Nacional de Salud, Lima, Peru. Analysis unit: Original articles including reports and series of cases completely developed in Peru or multicentre studies with participation of Peruvian settings. Interventions: Bibliographic search was performed in journals with indization in Medline, SciELO and LILACS 1981-2010. Year of publication, authors, time for realization, language, study area, publication type, study design, study field, population studied, type of research as defined by WHO, institutions participating, financing, and impact factor were registered for each article. Main outcome measures: Bibliometric indicators of production. Results: Two hundred and thirty-three articles on tuberculosis were identified. Average time of studies duration was $2.79 \pm 3.55$ years, and average time between end of study and publication was $2.95 \pm 1.65$ years. Study areas were $68(29.2 \%)$ mainly clinical, $66(28.3 \%)$ epidemiological, $56(24 \%)$ public health themes. Only $27.9 \%$ were published in a national biomedical journal. Most articles had participation of international institutions (71\%). Conclusions: In spite of evident increase in scientific production on TB in Peru, it is still far from the production of more developed countries. Along adopting measures to increase scientific production and research of TB as a national sanitary problem, it is necessary to prioritize such investigation by national agenda so it may be more effective.

Key words: Tuberculosis, bibliometrics, scientifific and technical publications, health priority agenda.

An Fac med. 2012;73(4):299-305

\section{INTRODUCCIÓN}

A nivel mundial, para el año 2010, se registraron 8,8 millones de casos incidentes de tuberculosis (TB), lo cual es equivalente a 128 casos por 100000 habitantes. Además, se estimó 1,1 millones de muertes por TB entre perso- nas VIH negativas y 350 mil muertes en personas con infección de VIH asociada. La mayoría de casos en el 2010 ocurrió en Asia (59\%) y África (26\%); solo 3\% de casos se dio en la región de las Américas ${ }^{(1)}$. En el Perú, para el año 2010, se atendió 32477 casos nuevos de TB; las regiones con tasas más altas fueron Lima, Callao, Ica, Tacna, Madre de Dios, Ucayali y Loreto. En dicho año se alcanzó una incidencia para el país de 96 casos por 100000 habitantes, mientras que la incidencia en el departamento de Lima llegó a 119 casos 
por 100000 habitantes, siendo una de las mayores de América Latina ${ }^{(1,2)}$. En Lima, la TB es un grave problema de salud pública; $86 \%$ de los casos de tuberculosis multidrogorresistentes (TBMDR) del país se encuentra en Lima y Callao; además, $86 \%$ de todos los casos de TB de Lima se encuentran concentrados en 18 de sus 43 distritos ${ }^{(2)}$. Esto evidencia que, a pesar que la morbilidad y mortalidad por tuberculosis ha disminuido en el Perú entre 1987 y el 2007 -producto de la transición epidemiológica ${ }^{(3)}$-, sigue siendo una prioridad sanitaria nacional ${ }^{(4)}$, por la persistencia del riesgo alto de enfermar de TB y TB MDR, y por la emergencia de formas graves y severas como la tuberculosis extremadamente resistente (TB $\mathrm{XDR})$, constituyendo una prioridad de investigación en salud ${ }^{(5,6)}$.

En el Perú, se ha desarrollado un Plan Estratégico Multisectorial de la Respuesta Nacional a la Tuberculosis para ser implementado en los siguientes 10 años. Dicho plan tiene como objetivo la disminución progresiva y sostenida de la incidencia y morbi-mortalidad por tuberculosis. De esta forma, para el año 2019, la incidencia de tuberculosis pulmonar frotis positivo debe ser menor de 40 casos por 100000 habitantes ${ }^{(6)}$. Sin embargo, dicho plan no puede ir desligado del desarrollo de investigación. Esto es evidente en el reporte del año 2011 del Control de la Tuberculosis de la Organización Mundial de la Salud, en el cual incluye un capítulo para Investigación y desarrollo ${ }^{(1)}$. Los progresos en el control de la TB están siendo limitados por estrategias, intervenciones y tecnologías viejas, cuya efectividad no está sustentada en evidencias, especialmente en países en desarrollo. Para lograr la eliminación de la TB, en el 2050, se requiere una transformación en prevención, diagnóstico y tratamiento de la TB. Para lograr esto, durante la década pasada se realizó grandes esfuerzos para desarrollar nuevos métodos diagnósticos, drogas y vacunas para $\mathrm{TB}$.

El total de fondos del 'Plan Global para detener la TB, 2011-2015' para financiamiento de investigación y desarrollo en TB fue de 614 millones de dólares americanos, en el año 2009; las principales fuentes de financiamiento fueron el Instituto Nacional de Salud de los Estados Unidos (NIH), la Fundación Bill y Melinda Gates, la Unión Europea, muchas otras agencias bilaterales o multilaterales y organizaciones filantrópicas. Actualmente, la investigación está dirigida al desarrollo de nuevos métodos diagnósticos de TB que permitan un diagnóstico simple, rápido, que pueda ser usado en establecimientos de salud del primer nivel y que permita diagnosticar tanto casos de TB como TB-MDR. Otro aspecto a desarrollar es el de nuevas drogas para tratamiento y prevención; es necesario tener drogas con esquemas cortos y simplificados, con mejores tasas de eficacia y tolerabilidad, para tratar TBMDR y casos de co-infección con VIH. También, se busca drogas que ayuden en el tratamiento de infección latente. El desarrollo de nuevas vacunas para prevención es otro campo de investigación vigente, junto con la investigación operativa, la cual es esencial para mejorar el cuidado y control de la TB y es útil para identificar las formas más efectivas de usar los recursos y herramientas disponibles ${ }^{(1)}$.

Los análisis bibliométricos evalúan en forma cuantitativa y cualitativa variables relacionadas a publicaciones científicas ${ }^{(7)}$. La producción científica sobre tuberculosis ha sido evaluada en estudios bibliométricos en España ${ }^{(8)}$ y Brasil ${ }^{(9)}$; a nivel nacional, no encontramos un estudio que analice a profundidad la producción científica en TB. Sin embargo, existe una experiencia previa para $\mathrm{VIH} /$ sida $^{(10)}$.

El objetivo de este estudio fue analizar las publicaciones relacionadas a TB realizadas en el Perú, en el periodo 1981-2010. Los resultados del presente análisis permitirán conocer el estado del arte de la investigación en el Perú sobre este importante problema de salud pública, las fortalezas y debilidades de dicha investigación, y permitir un alineamiento con la tendencia de in- vestigación y desarrollo a nivel mundial, teniendo como marco la realidad nacional.

\section{MÉTODOS}

La búsqueda bibliográfica fue realizada en MEDLINE, SciELO (regional y Perú), LILACS y LIPECS, entre mayo y junio de 2011, usando los portales web correspondientes (http://www.ncbi.nlm.nih.gov/pubmed/, http://www.scielo.org.pe/scielo.php, http://www.scielo.org/php/index. php, http://lilacs.bvsalud.org/es/ y http://www.bvs.org.pe/php/index.php). Dichas bases de datos fueron elegidas por su cobertura y rigurosidad científica para la inclusión de revistas biomédicas. Para complementar la búsqueda, se procedió con la revisión en Google Scholar. La estrategia de búsqueda para la recuperación de las publicaciones fueron 'tuberculosis' y 'Peru'. Se estableció como limites de búsqueda los años 1981 y 2010. Seleccionamos publicaciones desarrolladas en el Perú, o estudios multicéntricos con participación de sedes peruanas, independientemente de la nacionalidad de los autores. Solo se incluyó artículos originales, comunicaciones cortas; no entraron al análisis notas personales, cartas al editor, editoriales ni artículos de revisión.

Para cada artículo se registró las siguientes variables: año de publicación, autores, duración de la ejecución, idioma, área de estudio, tipo de publicación, diseño de estudio ${ }^{(11)}$, ámbito del estudio, población de estudio, tipo de investigación definida por OPS ${ }^{(12)}$, instituciones participantes, fuentes de financiamiento y factor de impacto de la revista empleando el Journal Citation Reports (JCR) del año 2010 (http:// scientific,thomson,com/products/jcr/).

Dichas variables fueron ingresadas a una base de datos en Excel MS 2007 para Windows, por dos médicos investigadores con experiencia en publicación de artículos científicos; además, se tuvo un control de calidad por un tercer investigador. Se realizó un análisis 
descriptivo (frecuencias y porcentajes); dichos resultados serán mostrados en tablas y gráficos.

\section{RESULTADOS}

Encontramos 233 artículos sobre tuberculosis en el Perú, publicados entre enero de 1981y diciembre de 2010. En el periodo 1981-1990 se publicó 10 artículos (4,3\%); en el periodo 1991 . 1995, 21 artículos (9,0\%); en el periodo 1996-2000, 18 artículos (7,7\%); en el periodo 2001-2005, 67 artículos (28,8\%); y, en el periodo 2006-2010, 117 artículos (50,2\%).

Del total de artículos, 174 fueron publicados en Pubmed, 23 en Scielo y 61 en LILACS; se publicó 19 artículos en dos bases de datos y tres artículos en las tres bases de datos, simultáneamente. En la figura 1, mostramos la tendencia en el número de publicaciones, según base de datos y año de publicación. A partir del año 2001, se encontró 184 (79\%) de las publicaciones en tuberculosis en las tres bases de datos analizadas. Mientras que en el periodo 1981-1990, solo se publicó 10 (4,3\%) artículos.

En 149 artículos (63,5\%) se pudo determinar el tiempo en años de duración del estudio y el tiempo entre la finalización del estudio y la publicación. El tiempo promedio de duración de los estudios fue de 2,79 \pm 3,55 años; 36 $(24,2 \%)$ artículos no duraron un año en su ejecución; $42(28,2 \%)$ artículos duraron un año; 49 (32,9\%), entre dos y cinco años; y $22(14,8)$ tomaron de seis a más años en su ejecución. El tiempo promedio entre la finalización del estudio y su publicación fue $2,95 \pm 1,65$ años. Solo cinco $(3,4 \%)$ artículos fueron publicados el mismo año de finalización, $23(15,4 \%)$ al año, 41 $(27,5 \%)$ a los dos años, $30(20,1 \%)$ a los tres años, $22(14,8 \%)$ a los cuatro años y $28(18,8 \%)$ de cinco a más años luego de su finalización.

Respecto al área de estudio del artículo, $68(29,2 \%)$ trataron principalmente temas clínicos, $66(28,3 \%)$ el

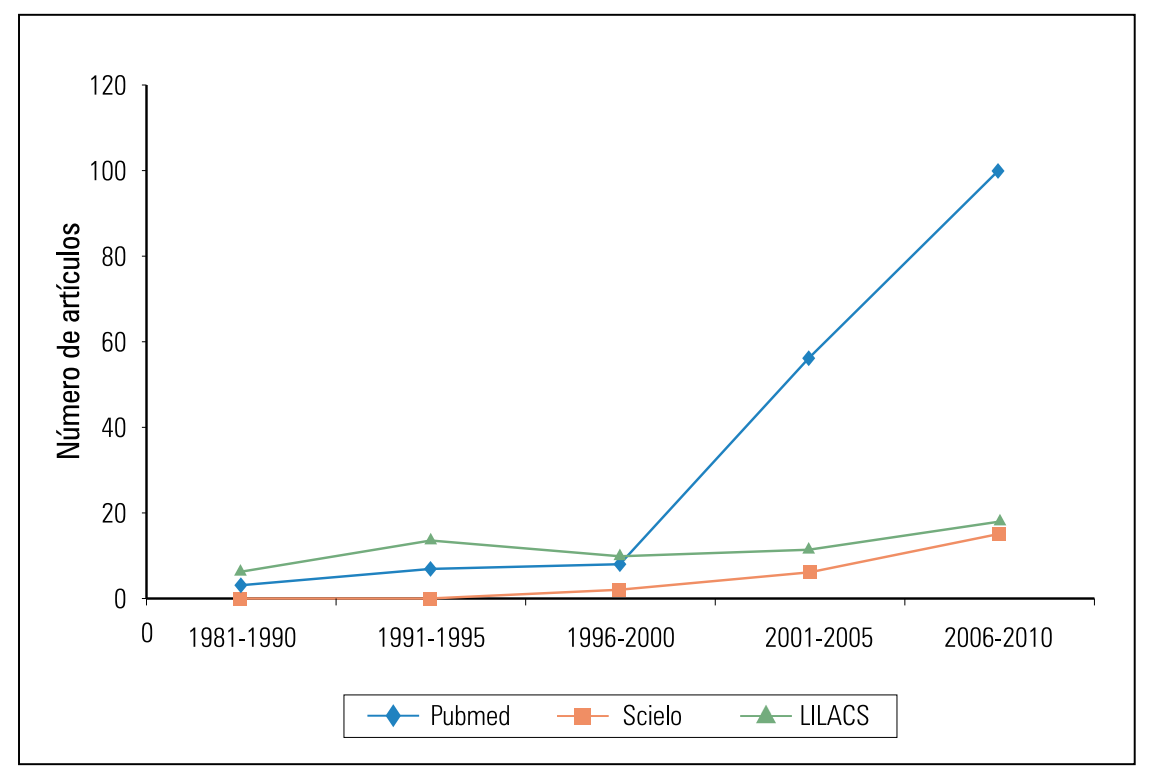

Figura 1. Número de artículos de tuberculosis en el Perú, periodo 1981-2010, según base de dato de publicación. área de epidemiologia, 56 (24\%) temas de salud pública, $23(9,9 \%)$ temas de ciencias básicas, 13 (5,6\%) gestión de servicios de salud y 7 (3\%) temas de economía de la salud. Según la clasificación de tipo de investigación de la OPS, 131 (56,2\%) artículos fueron investigación para conocer la enfermedad y factores de riesgo; 39 (16,7\%) fueron evaluaciones de impacto de interven- ciones actuales; 35 (15\%), evaluaciones de impacto de intervenciones nuevas; 21 (9\%) fueron investigaciones para sustentar inversiones en investigación en salud y medir el impacto y costo; seis $(2,6 \%)$ evaluaron costo-efectividad; y una $(0,4 \%)$ fue investigación operativa. La línea temática más explorada fue el estudio de diagnóstico de TB y TB-MDR, con 112 (48,1\%) artículos,
Tabla 1. Distribución de publicaciones de tuberculosis según tipo de diseño, en el periodo 1981-2010.

\begin{tabular}{lcc} 
Tipo de estudio & Número & Porcentaje \\
Artículo original & & \\
Transversal & 101 & 43,3 \\
Longitudinal & 31 & 13,3 \\
Casos y controles & 11 & 4,7 \\
Cohortes & 8 & 3,4 \\
Experimental (laboratorio) & 34 & 14,6 \\
Ensayo clínico controlado & 2 & 0,9 \\
Análisis costo beneficio & 6 & 2,6 \\
Análisis costo efectividad & 5 & 2,1 \\
Evaluación de impacto & 5 & 2,1 \\
Investigación operativa & 2 & 0,9 \\
Revisión sistemática / metaanálisis & 1 & 0,4 \\
Reporte de caso / serie de caso & 24 & 10,3 \\
\hline Total & $231^{*}$ & \\
\hline
\end{tabular}

* En dos artículos no se pudo identificar el tipo de estudio. 
seguida por tratamiento de TB y TBMDR con $48(20,6 \%)$ artículos, comorbilidad de TB con 24 (10,3\%), gestión de los servicios y programas de salud con $23(9,9 \%)$, políticas regionales en $\mathrm{TB}$ con nueve $(3,9 \%)$, prevención y educación en $\mathrm{TB}$ con seis $(2,6 \%)$, TB en población vulnerable con seis $(2,6 \%)$ y TB en trabajadores de salud con cinco $(2,1 \%)$ artículos.

En la tabla 1 mostramos los diseños de investigación usados en los artículos publicados. Encontramos que los estudios de tipo transversal fueron los más comunes, seguidos por los estudios de tipo longitudinal y experimentales de laboratorio. Los reportes y series de caso constituyeron el 10,3\% de artículos, mientras que solo encontramos una revisión sistemática.

Con respecto al ámbito de estudio o lugar donde fue realizada la investigación, 162 (73,3\%) artículos fueron realizados solo en Lima; 18 (8,1\%) fuera de Lima; 17 (7,7\%) en el Perú, como sede de un estudio multicéntrico internacional; 17 (7,7\%) investigaciones se llevaron a cabo en Lima y provincias con representatividad nacional y siete $(3,2 \%)$ fueron investigaciones realizadas en Lima y provincias sin representación nacional.

En el periodo 1981-1990, la producción científica fue limitada. No encontramos artículo de tuberculosis en el Perú sobre ciencias básicas, economía en salud y gestión de servicios. Dichas áreas temáticas recién fueron desarrolladas en artículos a partir del año 2001. Los artículos de ciencias básicas empezaron a ser publicados a partir del año 1991. Durante el último decenio, las áreas temáticas que más se han desarrollado en los artículos sobre tuberculosis en el Perú son Salud pública e intervenciones, epidemiologia y clínica (ver figura 2).

La mayoría de artículos fue publicada en idioma inglés (69,5\%); el restante consistió en artículos en español. Solo $27,9 \%$ de los artículos fue publicado en revistas biomédicas nacionales. En el periodo 1981-1990, solo tres artículos

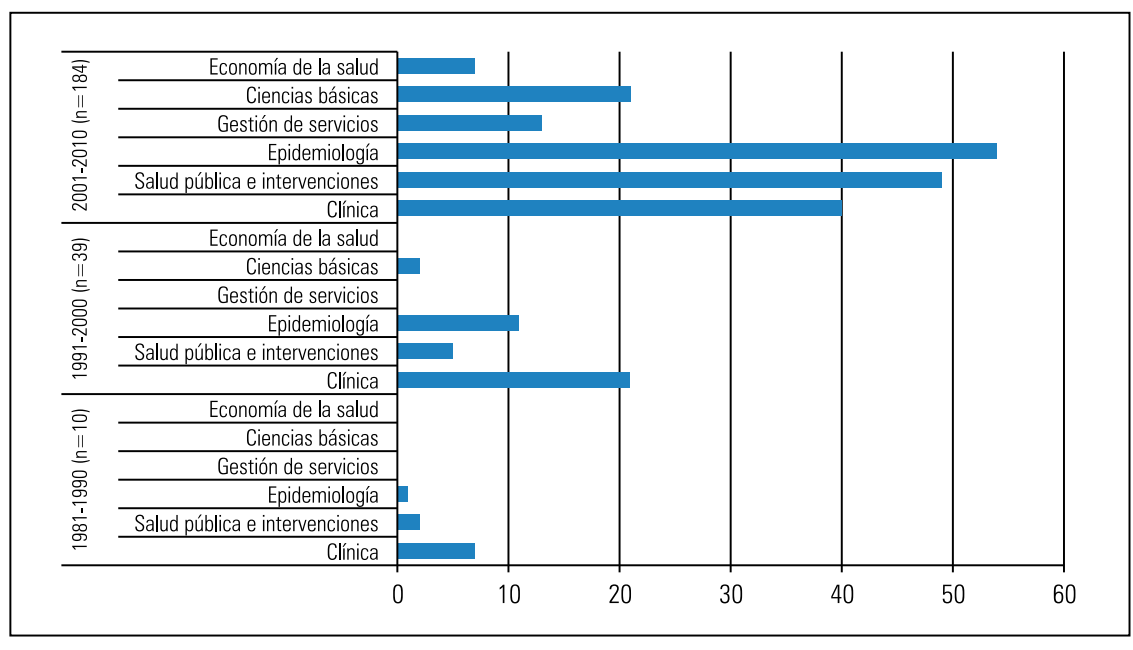

Figura 2. Área de investigación de artículos científicos sobre tuberculosis en el Perú, según periodos. fueron publicados en revistas internacionales; para el periodo 1991-1995 fueron cuatro artículos; en el periodo 1996-2000, ocho artículos; entre 2001. 2005, 53 artículos; y, entre 2006-2010, fueron 100 artículos publicados en revistas internacionales. Las revistas internacionales donde más se ha publicado artículos sobre tuberculosis en el Perú son The International Journal of Tuberculosis and Lung Disease, Journal of
Clinical Microbiology y Emerging Infectious Diseases; mientras que las revistas nacionales, donde más se ha publicado son Revista Peruana de Medicina Experimental y Salud Pública, Revista de Gastroenterología del Perú y Revista Médica Herediana. En total, los 233 artículos estudiados fueron publicados en 80 revistas biomédicas distintas, de las cuales solo 17 fueron revistas biomédicas peruanas. En la tabla 2, mostramos las
Tabla 2. Principales revistas biomédicas donde se publicó artículos sobre tuberculosis en el Perú, periodo 1981-2010.

\begin{tabular}{cccc} 
Revista & $\begin{array}{c}\text { Número de } \\
\text { artículos }\end{array}$ & Porcentaje & $\begin{array}{c}\text { Factor de } \\
\text { impacto }\end{array}$ \\
Int J Tuberc Lung Dis & 41 & 17,6 & 2,557 \\
Rev Peru Med Exp Salud Publica & 14 & 6,0 & N.D \\
J Clin Microbiol & 11 & 4,7 & 4,22 \\
Rev Gastroenterol Peru & 10 & 4,3 & N.D \\
Emerg Infect Dis & 9 & 3,9 & 6,85 \\
Rev Med Hered & 8 & 3,4 & N.D \\
Am J Trop Med Hyg & 8 & 3,4 & 2,44 \\
PLoS One & 6 & 2,6 & 4,41 \\
Lancet & 6 & 2,6 & 33,63 \\
N Engl J Med & 5 & 2,1 & 53.48 \\
AMIA Annu Symp Proc & 5 & 2,1 & N.D \\
Rev Peru Epidemiol & 4 & 1,7 & N.D \\
PLoS Med & 4 & 1,7 & 15,61 \\
Pediatrics & 4 & 1,7 & 5,39 \\
Bol Soc Peru Med Interna & 4 & 1,7 & N.D \\
\hline
\end{tabular}

N.D: no disponible 
15 primeras revistas en donde fueron publicados con más frecuencia los artículos.

En 200 artículos se pudo identificar las instituciones participantes: 65 $(32,5 \%)$ artículos fueron realizados por instituciones públicas y privadas nacionales e internacionales; 52 (26,0\%) por instituciones peruanas privadas e internacionales; 36 (18,0\%) por solo instituciones peruanas públicas; 14 (7,0\%) por solo instituciones internacionales; $14(7,0 \%)$ por instituciones públicas y privadas nacionales; 11 (5,5\%) por instituciones públicas nacionales e internacionales; y ocho $(4,0 \%)$ por instituciones privadas peruanas. Las 10 instituciones nacionales e internacionales con mayor participación en la investigación de tuberculosis en el Perú son mostradas en la tabla 3 .

\section{DISCUSIÓN}

El presente análisis bibliométrico muestra varios aspectos importantes de las publicaciones de tuberculosis en el Perú. El primer hallazgo es el incremento en la cantidad de artículos publicados a partir del año 2000, tanto en cantidad como en calidad. Existirían algunos indicadores de la mejora en la calidad de los artículos, como el mayor número de artículos publicados en revistas internacionales e indizadas en Pubmed, base de datos que presenta procesos que aseguran una mejor calidad de sus publicaciones. A pesar de que el incremento se da a partir del 2000 entre 2006 y 2010 el número de publicaciones en revistas internacionales se duplicó respecto al periodo 2000-2005; además, en los últimos cinco años se ha publicado la mitad de artículos del

Tabla 3. Principales instituciones nacionales e internacionales que publicaron sobre de tuberculosis en el Perú, periodo 1981-2010.

\begin{tabular}{lc} 
Institución & $\begin{array}{c}\text { Número de } \\
\text { publicaciones }\end{array}$ \\
\hline Públicas nacionales & 65 \\
\hline 1. Ministerio de Salud & 28 \\
2. Instituto Nacional de Salud & 20 \\
3. Hospital Nacional Dos de Mayo & 11 \\
4. Universidad Nacional Mayor de San Marcos (UNMSM) & 11 \\
5. Hospital Nacional Cayetano Heredia & 10 \\
6. Hospital Nacional Hipólito Unanue & 6 \\
\hline 7. Hospital Nacional Arzobispo Loayza & \\
\hline Privadas nacionales & 94 \\
\hline 8. Universidad Peruana Cayetano Heredia & 41 \\
9. Socios en Salud & 38 \\
10. Asociación benéfica Prisma & \\
\hline Internacionales & 42 \\
\hline 1. Universidad Johns Hopkins & 41 \\
2. Brigham and Women's Hospital & 38 \\
3. Imperial College London & 36 \\
4. Harvard Medical School & 27 \\
5. Partners in Health & 15 \\
6. Institute of Tropical Medicine Antwerp & 11 \\
7. U.S. Military HIV Research Program & 9 \\
8. The Centers for Disease Control and Prevention & 7 \\
9. Organización Mundial de la Salud & 6 \\
10. Massachusetts General Hospital & \\
\hline
\end{tabular}

periodo estudiado. Este fenómeno en el aumento de la producción científica en tuberculosis ha sido observado en un estudio bibliométrico realizado en Brasil en el periodo 1996-2006 ${ }^{(9)}$. Además del incremento en la producción científica, es importante destacar la mayor visibilidad de los artículos en las bases de datos, especialmente a partir del año 2000. La mayoría de artículos analizados fue recuperada de Pubmed. Dicha base de datos le otorga a los artículos una mayor visibilidad, especialmente si el artículo está publicado en una revista de difusión electrónica y con acceso libre. Es importante considerar que, a mayor número de bases enlazadas mayor sería el potencial de visibilidad de dichos artículos ${ }^{(13)}$. Por ende, no es menos importante que los artículos estén presentes en bases regionales como Scielo y LILACS; en este análisis solo 22 artículos fueron encontrados en más de una base de datos.

Es de destacar que las revistas donde más se ha publicado los artículos de tuberculosis en el Perú fueron The International Journal of Tuberculosis and Lung Disease, la cual tiene formato electrónico y acceso gratuito a sus números (disponible en: http://www.ingentaconnect.com/content/iuatld/ijtld), y The Journal of Clinical Microbiology, la cual necesita suscripción para el acceso a sus números. Un estudio bibliométrico de tuberculosis en España encontró que en dichas revistas internacionales fue donde más se publicó artículos de tuberculosis ${ }^{(8)}$. Es importante mencionar que dentro de las revistas donde más se ha publicado están tres revistas con un factor de impacto mayor de 10 (Lancet, $N$ Engl J Med y PLoS Med). Por otro lado, las revistas nacionales indizadas en Pubmed aún no cuentan con factor de impacto.

La duración promedio de las investigaciones fue de 2,8 años, debido probablemente al diseño de los estudios (transversales o longitudinales), los cuales en su mayoría requirieron dos o más años para su ejecución. Respecto al tiempo entre la finalización del estudio y la publicación, en promedio tomó 
casi tres años; las causas de este retraso se deberían a factores de los autores y de las revistas. Dentro de los factores de los autores estaría la demora en el análisis de los datos, demora en la preparación del manuscrito, desconocimiento de revistas para publicar, falta de interés en la publicación o retraso de los investigadores en responder las observaciones del comité editor. Por parte de los factores de las revistas, estarían la demora en el proceso editorial y la cantidad de artículos que la revista tiene para publicar, especialmente mayor en caso de revistas de alto factor de impacto alto ${ }^{(14)}$.

Con respecto al área de estudio, la mayoría de artículos fue sobre temas clínicos, epidemiológicos y de salud pública. Además, solo a partir del año 2001 hay artículos publicados sobre economía en salud y gestión de servicios de salud. Dicha tendencia también ha sido observada en un estudio bibliométrico de VIH/sida en el Perú ${ }^{(10)}$. Según la clasificación de tipo de investigación de la OPS, la mayoría de artículos consistió en investigaciones para conocer la enfermedad y factores de riesgo. Dicha investigación utilizó principalmente estudios de diseño transversal, longitudinal, casos y controles y cohortes, además de los reporte/series de caso. Ante este perfil en la investigación en tuberculosis en el Perú, es necesario fortalecer la investigación operativa, estudios costobeneficio, costo-efectividad y de impacto. Dichos estudios permiten llevar a cabo evaluaciones sistemáticas de las propiedades, efectos e impactos que tienen los actuales métodos diagnósticos, esquemas de tratamientos y programas de control y prevención de tuberculosis, con el objetivo de proveer información objetiva para apoyar la toma de decisiones y políticas de salud a nivel nacional, regional o local ${ }^{(15)}$. En Brasil, el perfil de diseños de investigación en tuberculosis es parecido, con predominancia de estudios descriptivos, transversales, caso-control y cohortes. Sin embargo, existe un mayor número de investigaciones operativas en el mismo periodo de tiempo ${ }^{(9)}$.
La línea temática más estudiada fue el diagnóstico de TB y TB-MDR y el tratamiento de TB y TB-MDR. Sin embargo, el enfoque de dichos temas fue descriptivo o establece asociaciones con algunos factores sociales, económicos, demográficos, entre otros. El desarrollo de estudios sobre gestión de servicios y programas de salud, políticas en TB, prevención y educación en TB, TB en población vulnerable y trabajadores de salud son una necesidad. Sin embargo, un primer paso para el desarrollo de investigación de acuerdo a las prioridades actuales es el establecimiento de una agenda de investigación basada en un proceso de priorización sistemático ${ }^{(16)}$. Dichos mecanismos facilitan el incremento armonizado de la investigación en salud, especialmente en el aspecto financiero y de impacto, en un ámbito donde los recursos económicos y humanos siempre son escasos. Un siguiente paso es el fortalecimiento de algunas capacidades para el desarrollo de las investigaciones con el objetivo de prevenir y controlar la tuberculosis en diversos ámbitos. Así por ejemplo, fortalecer las capacidades para el diseño e implementación de investigaciones operativas, ensayos clínicos y estudios pre-clínicos en el uso e incorporación de tecnologías de la información y las comunicaciones (TIC) en la prevención y control de TB, de manera de planificar y evaluar las intervenciones en salud pública, entre otros aspectos.

La mayoría de artículos tuvo participación de alguna institución internacional (71\%); en un estudio bibliométrico sobre VIH/sida en el Perú, dicha participación llegó al 54,3\% de artículos ${ }^{(10)}$. Dentro de las instituciones nacionales, la Universidad Peruana Cayetana Heredia (UPCH) es la que presentó mayor participación, seguida por el Ministerio de Salud. Dos instituciones privadas, como Socios en Salud y la Asociación Benéfica Prisma, tuvieron una gran participación, mientras que el Instituto Nacional de Salud fue la quinta institución en tomar parte en investigación en tuberculosis. Dichas instituciones también son las que tie- nen mayor participación en las investigaciones en VIH/sida en el Perú ${ }^{(10)}$. Respecto a las instituciones internacionales, la Universidad Johns Hopkins, el Brigham and Women's Hospital, el Imperial College London, la Escuela de Medicina de Harvard y Partners in Health son las instituciones de mayor participación desde 1984. Es necesario esclarecer que Socios en salud es la sede peruana de Partners in Health, por lo que su aporte es la segunda más importante. Además, dicha institución trabaja en forma colaborativa con el Brigham and Women's Hospital y la Universidad de Harvard. El importante aporte de la UPCH es facilitado por el desarrollo de investigación conjunta con dichas instituciones extranjeras, entre otras. De esta forma, se destaca la importancia de la investigación colaborativa, lo cual se ve reflejado en una mayor producción científica.

Encontramos diferencias con la participación de instituciones internacionales en la investigación de $\mathrm{VIH} /$ sida, donde la Universidad de Washington, la Unidad de Investigación de la Marina de los EE UU y la Universidad de California fueron las más importantes ${ }^{(10)}$. Dichas diferencias podrían deberse a que la investigación priorizada por dichas instituciones ha sido establecida según intereses específicos de los investigadores principales o líderes de dichas instituciones. Al igual que con los artículos sobre VIH/sida, la investigación universitaria tiene a la UPCH y la UNMSM como las principales productoras ${ }^{(10)}$. Es importante destacar la participación activa de los hospitales del Ministerio de Salud en la investigación en tuberculosis, especialmente de los Hospitales Dos de Mayo, Cayetano Heredia, Hipólito Unanue y Arzobispo Loayza. Sus aportes en estudios clínicos, epidemiológicos y reportes/series de casos han sido significativos en el desarrollo del conocimiento de esta enfermedad en nuestro país.

Las limitaciones del estudio están relacionadas a las bases de datos utilizadas en la búsqueda. Incluimos Pubmed, Scielo y LILACS, pues dichas bases re- 
cuperan la mayor cantidad de artículos sobre tuberculosis realizada en nuestro país. Para el presente análisis, solo consideramos artículos originales y reportes de casos; no se incluyó tesis, artículos de revisión ni cartas al editor, por lo que el número de artículos fue reducido durante el análisis. Tampoco incluimos en el análisis el número de autores, el país de la institución participante y financiantes. Por la naturaleza el estudio, está sujeto al sesgo de publicación, ya que las bases examinadas no tienen registradas toda la producción científica sobre tuberculosis realizada en el Perú, además que muchas investigaciones no llegan a publicarse, sobre todo aquellas desarrolladas por instituciones públicas nacionales, como hospitales o universidades del interior del país. El periodo estudiado fue establecido arbitrariamente, publicaciones anteriores a dicho periodo fueron de difícil acceso y su número es pequeño, no contribuyendo con el análisis. Respecto al factor de impacto, al ser un indicador que varía año tras año, solo consideramos el factor de impacto del 2010, para brindar un panorama general de las características de las revistas donde los artículos son publicados.

La producción científica sobre tuberculosis en el Perú se ha incrementado en los últimos diez años. Sin embargo, sigue siendo menor comparada a la producción de Brasil, que en el mismo periodo de tiempo publicó 388 artículos ${ }^{(9)}$, y España, que entre 1997 y el 2006 publicó 1191 artículos ${ }^{(8)}$. Estas diferencias se deberían a la desproporcionalidad que existe en el porcentaje asignado del producto bruto interno al desarrollo e investigación, que para el año 2007 fue 1,1\% en Brasil y 1,27\% en España. En el Perú, dicho porcentaje apenas llega al 0,15\% ${ }^{(17)}$. Sin embargo, además de fortalecer la investigación en tuberculosis en nuestro país, con el afán de incrementar la productividad científica, es igual de importante guiar dicha investigación en su contenido, mediante el establecimiento de una agenda de investigación en tuberculosis que fortalezca la investigación operativa, estudios de impacto de intervenciones nuevas y actuales, y estudios de costo-efectividad, costo beneficio. Los resultados de este análisis brindan importantes hallazgos; el primero muestra que si bien nuestra productividad es baja tiene un tendencia creciente en los últimos 5 años; segundo, que los artículos de tuberculosis en el Perú han sido realizados para la descripción de la infección en cuanto a tratamiento y diagnóstico mediante estudios clínicos y epidemiológicos; y, tercero, existen núcleos de investigación en tuberculosis que producen la mayoría de artículos mediante cooperación con instituciones internacionales también conocidas. Dicho conocimiento servirá como línea de base para la elaboración de un mecanismo de priorización que refuerce y oriente efectivamente el contenido de una agenda de investigación en tuberculosis, la cual debería ir acompañada de financiamiento nacional o internacional. Es probable que la agenda de investigación siga la tendencia actual de investigación en $\mathrm{TB}$, que incluye la investigación de nuevos métodos diagnósticos de TB, el desarrollo de esquemas cortos y simplificados y el desarrollo de investigación operativa que ayude a mejorar el uso de los escasos recursos para el cuidado y control de la TB en el Perú.

\section{REFERENCIAS BIBLIOGRÁFICAS}

1. WHO report 2011. Global Tuberculosis Control Disponible en: http://www.who.int/tb/publications/ global_report/2011/gtbr11_full.pdf

2. Bonilla C. Situación de la tuberculosis en el Perú Acta Med Per. 2008;25(3):163-70.

3. Análisis de la situación de salud en el Perú. 2010. Ministerio de Salud del Perú. Dirección General de Epidemiologia. Disponible en: http://www.dge.gob. pe/publicaciones/pub_asis/asis25.pdf

4. Plan Nacional Concertado de Salud. 2007. Ministerio de Salud del Perú

5. Caballero P, Yagui M, Espinoza M, Castilla T, Granados A, Velásquez A y col. Prioridades Regionales y Nacionales de Investigación en Salud, Perú 2010 - 2014: un proceso con enfoque participativo y descentralista. Rev peru med exp salud publica. 2010;27(3):398-411.
6. Plan estratégico Multisectorial de la Respuesta Nacional a la Tuberculosis en el Perú 2010-2019. Ministerio de Salud. Dirección General de Salud de las personas. 2010.

7. Bibliometric handbook for Karolinska Institutet. Catharina Rehn and UIf Kronman, Karolinska Institutet University Library. Version 1.05, 2008.

8. Ramos J, Masiá M, Padilla S, Garcia-Pachón E, Gutiérrez F. Spanish scientific research output on tuberculosis indexed in MEDLINE, 1997-2006. Arch Bronconeumol. 2009;45(6):271-8.

9. Lineu A, Scatena T, Trajman A, Lapa J, Medronho R, Ruffino A. Two decades of research on tuberculosis in Brazil: state of the art of scientific publications. Rev Saúde Pública. 2007;41(Suppl.1):9-14.

10. Caballero P, Gutiérrez C, Rosell G, Yagui M, Alarcón $\mathrm{J}$, Espinoza M y col. Análisis bibliométrico de la producción científica sobre VIH/SIDA en el Perú 1985 - 2010. Rev peru med exp salud publica. 2011;28(3):470-6.

11. Rothman K, Greenland S. Modern Epidemiology. 2da Ed. Filadelfia: Lippincott Williams \& Wilkins; 1998.

12. Instituto Nacional de Salud. Guía del facilitador. Proceso de definición de prioridades nacionales de investigación en salud. Lima: Ministerio de salud; 2009.

13. Huamani Ch, Pacheco J. Visibilidad y producción de las revistas biomédicas peruanas. Rev Gastroenterol Perú. 2009;29-2:132-9.

14. Alarcón J, Romaní F, Gutiérrez C. Publicaciones científicas estudiantiles producidas en el curso de Epidemiología de la Facultad de Medicina de la Universidad Nacional Mayor de San Marcos durante el periodo 2003-2009. An Fac med. 2010;71(2):111-6.

15. International Network of Agencies for Health Technology Assessment (INAHTA). Health Technology Assessment (HTA) Glossary.1st ed. Stocklom, INAHTA; 2006.

16. Curisinche M, Yagui M, Castilla T, Cabezas C, Escalante G, Casas M, Lucero J. Proceso de construcción de la agenda nacional de investigación sobre Recursos Humanos en Salud (RHUS) en el Perú, 2011-2014. Rev peru med exp salud publica. 2011;28(2):372-81.

17. UNESCO SCIENCE REPORT 2010. The current Status of Science around the world. UNESCO Publishing. Second revised edition.

Articulo recibido el 12 de junio de 2012 y aceptado para publicación el 4 de setiembre de 2012.

Fuente de Financiamiento:

Instituto Nacional de Salud

Conflictos de Interés:

Los autores participaron en el diseño, implementación y evaluación de este proceso

\section{Correspondencia:}

Dr. Martín Yagui Moscoso

Dirección: Cápac Yupanqui 1400, Lima 11, Perú.

Teléfono: (511) 617-6200

Correo electrónico: myagui@ins.gob.pe 